\title{
An algorithm for the detection of low frequency solar radio burst
}

\begin{abstract}
Background: In this work, we develop a simple algorithm which detect major solar flares reliably and the amount of false detection cases was limited. The radio frequencies below $1000 \mathrm{MHz}$ are allocated for various services. However, this frequency range also has interesting scientific aspects. The Sun is very active also these frequencies, e.g. wellknown solar II and III type bursts appear in these frequencies. It is challenging to separate interference signals from real solar radio burst since these signals have quite often similar features.
\end{abstract}

Keywords: solar radio burst, radio frequencies, separate interference signals, solar flares
Volume 4 Issue 3 - 2020

\author{
Juha Kallunki \\ Metsähovi Radio Observatory, Aalto University, Finland
}

Correspondence: Juha Kallunki, Metsähovi Radio

Observatory, Aalto University, Metsähovintie I I 402540 Kylmälä,

Finland, Tel +358 29 4424852, Email juha.kallnki@aalto.fi

Received: June 09, 2019 | Published: June 22, 2020
Abbreviations: RAS, radio astronomy services; RFI, radio frequency interference; CFAR, constant false alarm rate; FAR, false alarm rate; $\mathrm{MRO}$, metsähovi radio observatory

\section{Introduction}

The detection of the low frequency, frequencies below $1000 \mathrm{MHz}$, solar radio burst is challenging since that frequency range is heavily filled with various radio transmitters and other electronic devices, which are producing interference on the radio wavelength regime. Only minor part of this band is allocated to passive services, as such as radio astronomy services (RAS). The most common and well-known transmissions are broadcasts and mobile phones' up and down links. In addition, several electronic devices as such as switching power supplies and lighting solutions are causing interference: conductive and emitting. Each observatory has own Radio Frequency Interference (RFI) environment. The technical possibilities for filtering out unwanted signals are rather limited. For instance, some notch filters are possible, but it is a risk that we will miss some important bands using these, since filters rejection bands are not ideally matched. The solutions should be designed according the individual needs. It is rather difficult to design generic solution which could be work even satisfactory in all locations. Continuous and weak interference signals are relatively simple to identify and separate from solar flares. However, some single interference signals have a very similar pattern (e.g. drifting structure) than in regular solar flares. That is why inclusive automatic detection is almost impossible. Several detection algorithms and methods have been developed in the recent years. ${ }^{1,2,3}$ In this article, we will present a simple model which could reliable detect strong and wide-frequency range solar radio bursts. In addition, the amount of the false detection is limited. Amount is so small, that they can be visually inspected within a reasonable time frame.

The main idea is to find automatically and reliably scientific interesting flares without having too much false positive detection. Some author use term Constant False Alarm Rate (CFAR) or False Alarm Rate $(\mathrm{FAR})^{1}$ for this. Some low energy solar radio burst will be missed; however, their scientific significance is rather low. In this work, we do not focus to identify or classify solar bursts according to their types (e.g. types II or III). Aalto University Metsähovi Radio Observatory (MRO) is situated in the Southern Finland GPS: N 60:13.04, E 24:23.35), some $45 \mathrm{~km}$ from the capital, Helsinki. The nearest bigger village (Kylmälä) is around $10 \mathrm{~km}$ away and the nearest settlements lie at a $550 \mathrm{~m}$ distance from the observatory. Thus, the location of the observatory is not an ideal for the low-frequency radio observations. ${ }^{6} \mathrm{MRO}$ is a node $\mathrm{e}^{4,5}$ of the worldwide solar radio burst-observing network, e-Callisto ${ }^{7}$ since the fall of 2010. The network is coordinated by ETH Zurich, and it consists of more than 170 instruments in different locations all over the world, covering wideband solar radio observations 24 hours a day throughout the year. The network focuses on the decimeter and meter wavelength range. A current frequency coverage at MRO is $16-850 \mathrm{MHz}$.

\section{Automatic solar radio burst detection}

In this work, the automatic solar burst detection algorithm was tested with e-Callisto data on frequency range between $45 \mathrm{MHz}$ and $845 \mathrm{MHz}$. e-Callisto system produce every 15 minutes a new FIT file from the sunrise to sunset. The detection algorithm was applied for each FIT file. Automatic low frequency solar radio burst alarming system is a trigger for other MRO's radio telescopes and instruments that they can move to the solar observing mode after we have got a notification from low solar radio instrument. Also, we wanted to keep the detection system as a simple as possible. For instance, we tried to keep number of fixed threshold values as low as possible. Usually, simpler algorithms are feasible for updates and maintenance. In Figure 1 , a simplified flow chart about the detection process. Our detection method has three phases. In the first phase, the data is filtered with an average filter. This removes effectively all the continuous interference signals, e.g. broadcast signals which could be seen in Figure 2 (upper left panel). The effect of average filtering is shown in Figure 2 (upper right panel). The second stage is that data is converted to the binary form. All the values, which are ten units above background level are marked as " 1 ", otherwise data point value is marked as " 0 ". The cut of value (ten units) is founded on experiential tests.

The binary conversion removes weak interference signals. There is small probability that in this conversion we will lose some weak solar radio flares, however those scientific impact is minor. In Figure 2 (lower left panel), the effect of the binary conversion is shown. In the third data processing stage, $3 \mathrm{D}$-spectrum is converted to $2 \mathrm{D}$-spectrum so that all the data points are integrated together in each time stamp (Figure 2, lower right plot). Finally, the actual inspection will be made from $2 \mathrm{D}$-spectrum with following criteria: 


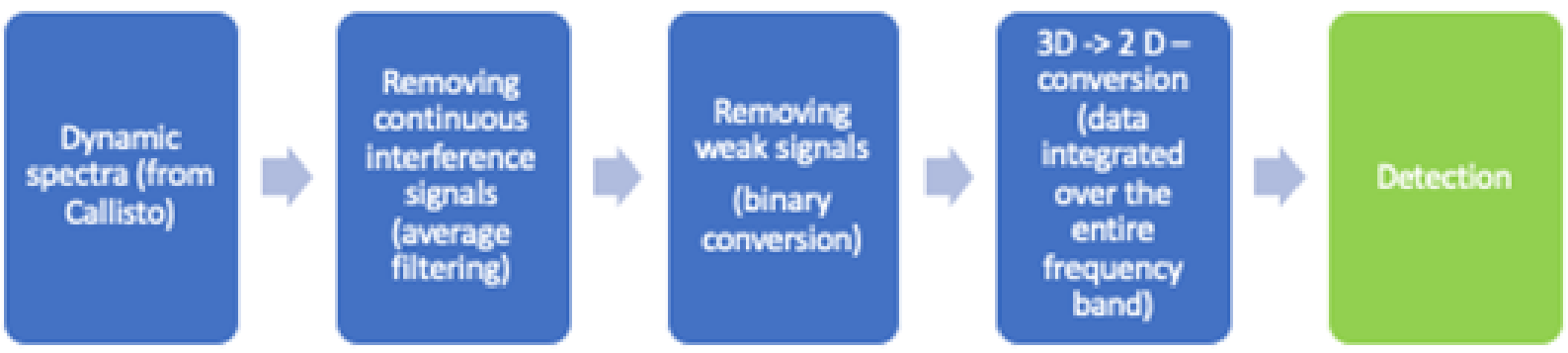

Figure I A simplified flow chart about the detection process.
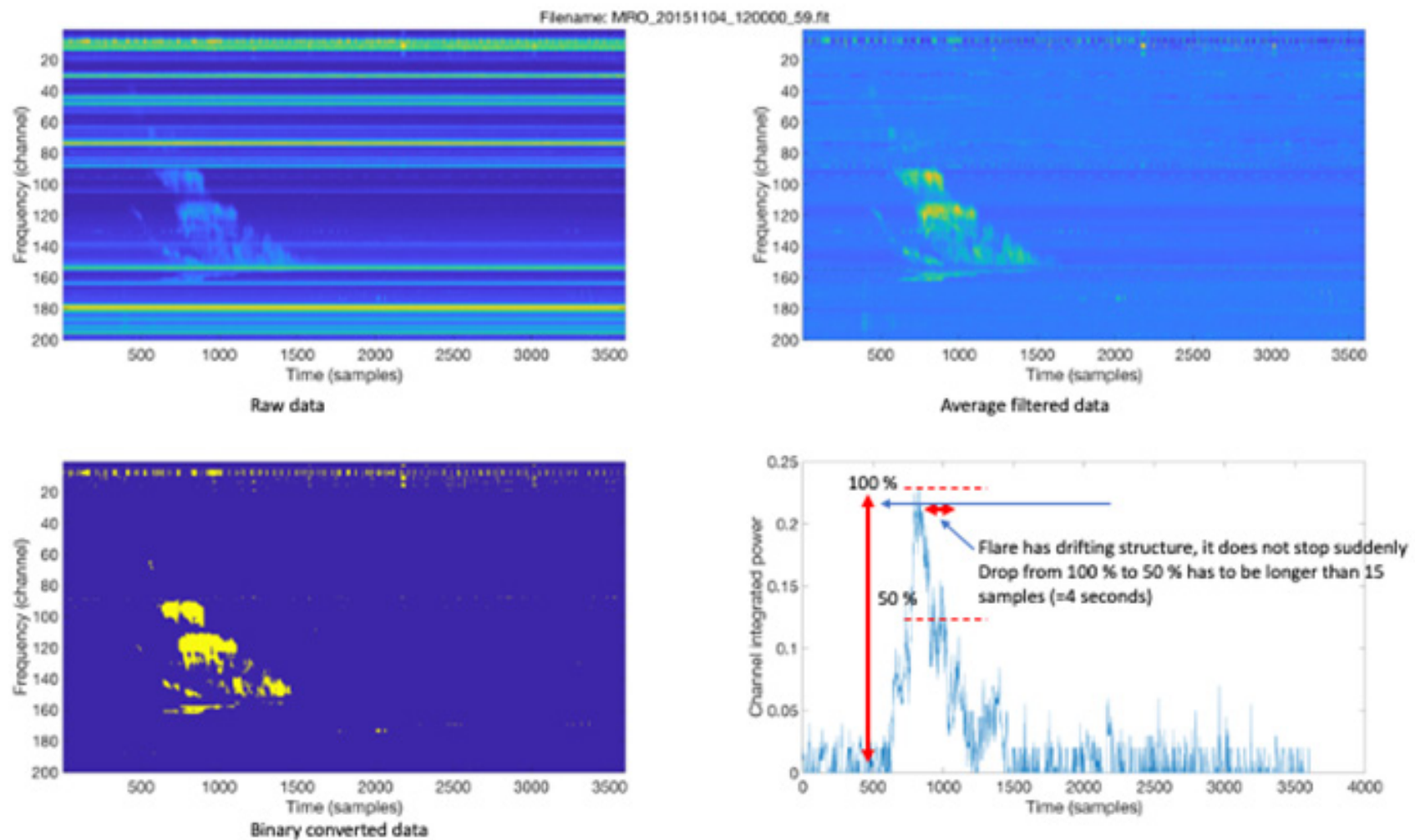

Figure 2 In upper left panel, the original dynamic spectra; in upper right panel, average filtered dynamic spectra; in the lower left panel, binary converted dynamic spectra and in lower right panel, 2D-spectrum where all data points are integrated together in each time stamp. In these dynamic spectra, we will see a typical type II solar radio burst.

1. The radio burst has to be $120 \mathrm{MHz}$ wide or more.

a. This will eliminate many narrow band interferences.

2. The burst has to be drifting or physical structure. The signal cannot suddenly light up or down from its full wide (detailed explanation is shown in Figure 2, lower right panel).

a. This will eliminate sharp and short-period spikes (e.g. lightning strikes) or sudden interference signals.

3. Other detections are classified as a possible solar radio burst.

The algorithm was developed and tested with Matlab. For further use, algorithm will be converted to Python, since it can be run more effectively from Unix environment. The detection software, for instance, could be run as a Unix cronjob on a daily basis.

\section{Results}

We analyzed all the data years between 2011 and 2019. In general, the solar activity was rather low on that time. The Solar Cycle 24 was weaker cycle compared with earlier cycles. Totally, we found 93 events, and 28 of them proved to be real solar radio event. 55 events were false positives and ten events were test cases. In test cases, artificially interference signals were created intentionally. Almost all the false positive detections were solar radio burst-like event. A few of these events have presented in Figure 3. We can notice that these events have similar features than in regular solar radio bursts. These interference signals are wide enough in frequency range and, in addition they have a drifting structure. 

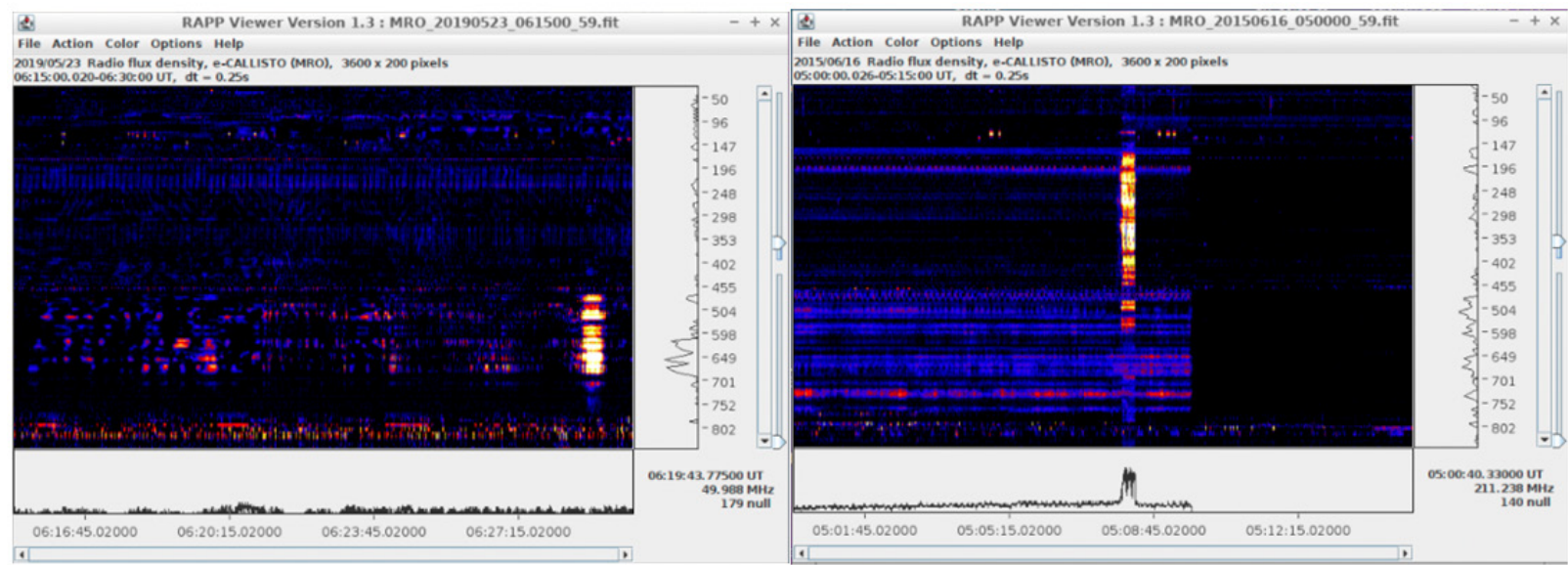

Figure 3 Two examples of false positive signals which were detected automatically. These interference signals have similar features with a regular solar flare.

\section{Conclusion}

The main aim for this detection was that we could reliably detect major, scientific interesting solar radio bursts and events. Without any automatic detection method, several solar radio bursts could be totally missed. This goal was achieved. In addition, the amount of false detection cases was limited, which are possible to investigate visually later on. The algorithm could be improved, thus is it could detect weaker solar radio bursts. This implementation needs further investigations. However, the weak solar radio events have rather limited scientific value on the field of solar radio astronomy.

\section{Acknowledgments}

None.

\section{Conflicts of interest}

Authors declare that there is no conflict of interest.

\section{Funding}

None.

\section{References}

1. Salmane H, Weber R, Abed-Meraim K, et al. A method for the automated detection of solar radio bursts in dynamic spectra. J Space Weather Space Clim. 2018;8:18.
2. Singh D, Raja KS, Subramanian P, et al. Automated Detection of Solar Radio Bursts using a Statistical Method, Solar Physics. 2019;294:112.

3. Zhang PJ, Wang CB, Ye L. A type III radio burst automatic analysis system and statistic results for a half solar cycle with Nançay Decameter Array data, $A \& A$. 2018;618.

4. Kallunki J, Monstein C, Uunila M. Callisto radio spectrometer for observing the Sun -Metsähovi Radio Observatory joins the worldwide observing network. IEEE Aerospace and Electronic Systems Magazine, 2013. p. 5-9.

5. Kallunki J, Uunila M, McKay-Bukowski D. Multi-frequency solar observations at Metsähovi Radio Observatory and KAIRA. Astronomische Nachrichten. 2015;336(6):566.

6. Kallunki J, Bezrukov D, Avotins V, et al. Particularly Low-Cost Portable Radio Frequency Interference Monitoring System. International Journal of Electromagnetic (IJEL). 2019;2(1):1-7.

7. e-Callisto. International Network of Solar Radio Spectrometers, a Space Weather Instrument Array. 2020. 